\title{
Agenesia de vena cava inferior en un caso de autopsia forense
}

\section{Absence of the inferior cava vein in a case of forensic autopsy}

\begin{abstract}
Resumen
La agenesia de vena cava inferior es una entidad congénita poco frecuente que a menudo se asocia con otras severas anomalías congénitas o malposiciones viscerales. También ha sido descrita como un factor de riesgo de trombosis venosa en pacientes jóvenes. La mayoría de los casos publicados proceden de estudios radiológicos y muy raramente aparece como hallazgo necrópsico. Presentamos un caso de agenesia de vena cava inferior, sin otras anomalías asociadas, que apareció como hallazgo incidental en una autopsia forense.
\end{abstract}

Palabras clave: Agenesia de vena cava inferior. Hallazgo incidental. Autopsia forense.

\section{Abstract}

The absence of the inferior vena cava is a rare congenital anomaly, often associated with severe congenital deformities and visceral position abnormalities. It has been described also as a risk factor for deep vein thrombosis in young patients. We present a case of congenital absence of inferior vena cava appeared as an incidental finding in a forensic autopsy.

Key words: Congenital absence of inferior vena cava. Incidental finding. Forensic autopsy.

\section{Introducción}

La agenesia de vena cava inferior es una entidad congénita poco frecuente que a menudo se asocia con otras anomalías congénitas severas o malposiciones viscerales. También ha sido descrita como factor de riesgo de trombosis venosa en pacientes jóvenes. La mayoría de los casos publicados proceden de estudios radiológicos y sólo raramente aparece como hallazgo necrópsico. Presentamos un caso de agenesia de vena cava inferior, sin otras anomalías asociadas, que se observó como hallazgo incidental en una autopsia forense.

\section{Presentación del caso}

Varón de 57 años que vivía solo y fue hallado muerto con abundantes restos de sangre en el suelo del dormitorio y en el pasillo del domicilio. Se encontró además una entrada del glucómetro de cuatro días previos al levantamiento con una glucemia de $532 \mathrm{mg} / \mathrm{dL}$.

Presentaba los siguientes antecedentes personales de interés:

- Exéresis quirúrgica de pólipo gástrico hiperplásico.

- Retraso mental moderado.

- Anemia ferropénica.

- Cirrosis hepática por virus de la hepatitis C sin descompensaciones.

- Hipertensión arterial en tratamiento con enalaprilo.

- Diabetes mellitus tipo II en tratamiento con insulina y metformina (Biguanida). Realizaba un deficiente control metabólico lo que había motivado múltiples consultas a urgencias por hiperglucemias simples.

\author{
M. Subirana Domènech \\ I. Galtés Vicente \\ G. Font Valsecchi \\ A. Xifró Collsamata
}

Médicos Forenses. Instituto de Medicina Legal de Catalunya. Subdirección de Barcelona.

Correspondencia: Dra. Mercè Subirana Servicio de Patología Forense. Zona Sur. Institut Medicina Legal de Catalunya. Ciutat de la Justícia. Edificio G. Gran Vía Corts Catalanes, 111 08014 Barcelona E-mail: 25402msd@comb.cat

Fecha de recepción: 10.FEB.2011

Fecha de aceptación: 21.FEB.2011 
Ingresó en el hospital un mes antes de la defunción para estudio de síndrome tóxico con pérdida ponderal no cuantificada. Se practicó endoscopia digestiva alta y baja que no evidenció la causa el cuadro anémico.

\section{Hallazgos de autopsia}

El cadáver se encontraba en fase cromática de putrefacción con mancha verde abdominal, torácica y cervical. Había restos de sangre coagulada en cara, parte anterior de piernas y plantas de los pies. El origen del sangrado se localizó en fosas nasales, sin fractura nasal subyacente.

En el examen interno se apreció una agenesia de vena cava inferior sin otras malformaciones congénitas o malposiciones viscerales asociadas. Las venas suprahepáticas drenaban directamente en aurícula derecha (Figuras 1 y 2). Hígado de peso en el límite inferior de la normalidad (1.380 gramos) con patrón de cirrosis micronodular y congestión periférica de los sinusoides. Pulmones de peso 780 derecho y 820 izquierdo con patrón de condensación bilateral difusa. En el resto de órganos, la autopsia no puso de manifiesto otras alteraciones, a excepción de cambios autolíticos generalizados.

El estudio histopatológico evidenció una bronconeumonía aguda severa, cirrosis hepática en grado marcado y prostatitis focal crónica, así como cambios relacionados con la autolisis.

El análisis toxicológico en orina puso de manifiesto etanol $(0.1 \mathrm{~g} / \mathrm{L})$. El análisis bioquímico puso de manifiesto una glucosuria de $359 \mathrm{mg} / \mathrm{dL}$ sin cuerpos cetónicos.

Teniendo en cuenta los datos obtenidos en el levantamiento (entrada en el glucómetro de una glucemia de $532 \mathrm{mg} / \mathrm{dL}$ ), autopsia y de estudios complementarios, se estableció como causa de muerte más probable un coma diabético secundario a diabetes mellitus tipo II descompensada por una bronconeumonía aguda severa.

\section{Discusión}

La agenesia de la vena cava inferior es una malformación congénita poco frecuente y la mayoría de los casos publicados se refieren a casos pediátricos asociados a cardiopatía congénita cianosante ${ }^{1}$. En las escasas series referidas a adultos suele ser una anomalía asintomática en el 70\% de los casos y aparece como un hallazgo incidental radiológico ${ }^{2}$ relacionado con el aumento en la realización de TC y cateterismos cardíacos. Más raramente aparece como hallazgo autópsico y quizás éste sea el motivo de que la iconografía sea escasa.

Cuando cursa clínicamente suele ser como insuficiencia venosa de extremidades inferiores y como factor de riesgo de trombosis venosa profunda uni o bilateral de los miembros inferiores, generalmente ilíaca en jóvenes sin trastornos de la coagulación ${ }^{3,4,5,6,7}$ o tromboembolismo pulmonar ${ }^{8}$. En algún caso, esta entidad ha simulado una tumoración mediastínica ${ }^{9,10,17}$ o ha debutado como una forma atípica de abdomen agudo ${ }^{11,12}$

La vena cava inferior tiene un desarrollo embrionario complejo ya que es el resultado de la aparición y regresión de tres pares de venas embrionarias ${ }^{13} \mathrm{y}$, por tanto, son múltiples las posibilidades de malformaciones congénitas a este nivel ${ }^{14,15}$. Se han publicado hasta 63 tipos de malformaciones, siendo las más frecuentes: transposición, duplicidad, agenesia de la vena renal izquierda retroaórtica (regresión del arco anterior del anillo renal y desarrollo del posterior), vena renal izquierda doble (rodeando la aorta: persistencia de la rama dorsal del riñón izquierdo y de la anastomosis inter supracardinal) ${ }^{16,17,18}$. A pesar de que se asocia a otras malformaciones, principalmente de tipo cardiovascular en un $40 \%$ : dextrocardias, comunicación interauricular, estenosis de arteria pulmonar, también con la transposición de vísceras abdominales, disgenesia pulmonar, poliesplenia o asplenia ${ }^{6}$, o formando parte del síndrome de Ivemark (agenesia esplénica, corazón bilobulado y situs inversus) $)^{19}$, también se puede presentar, como en nuestro caso, sin ningún hallazgo patológico asociado. A pesar de que la agenesia de vena cava inferior que hemos presentado no fue responsable del fallecimiento, consideramos que la difusión de su imagen ayuda a reconocer visualmente esta variante anatómica escasamente ilustrada en los textos de patología forense.

\section{Agradecimientos}

Al Dr. Juan Carlos Borondo y Dra. Susana Moyano Corvillo de la sección de Histopatología del INT y CF (Barcelona), al facultativo Agustí Mora y la Dra. Amparo Arroyo, jefa de Servicio del Laboratorio del IMLC, por su colaboración en las pruebas complementarias realizadas. A los técnicos del servicio de Patología por su dedicación y entusiasmo. 

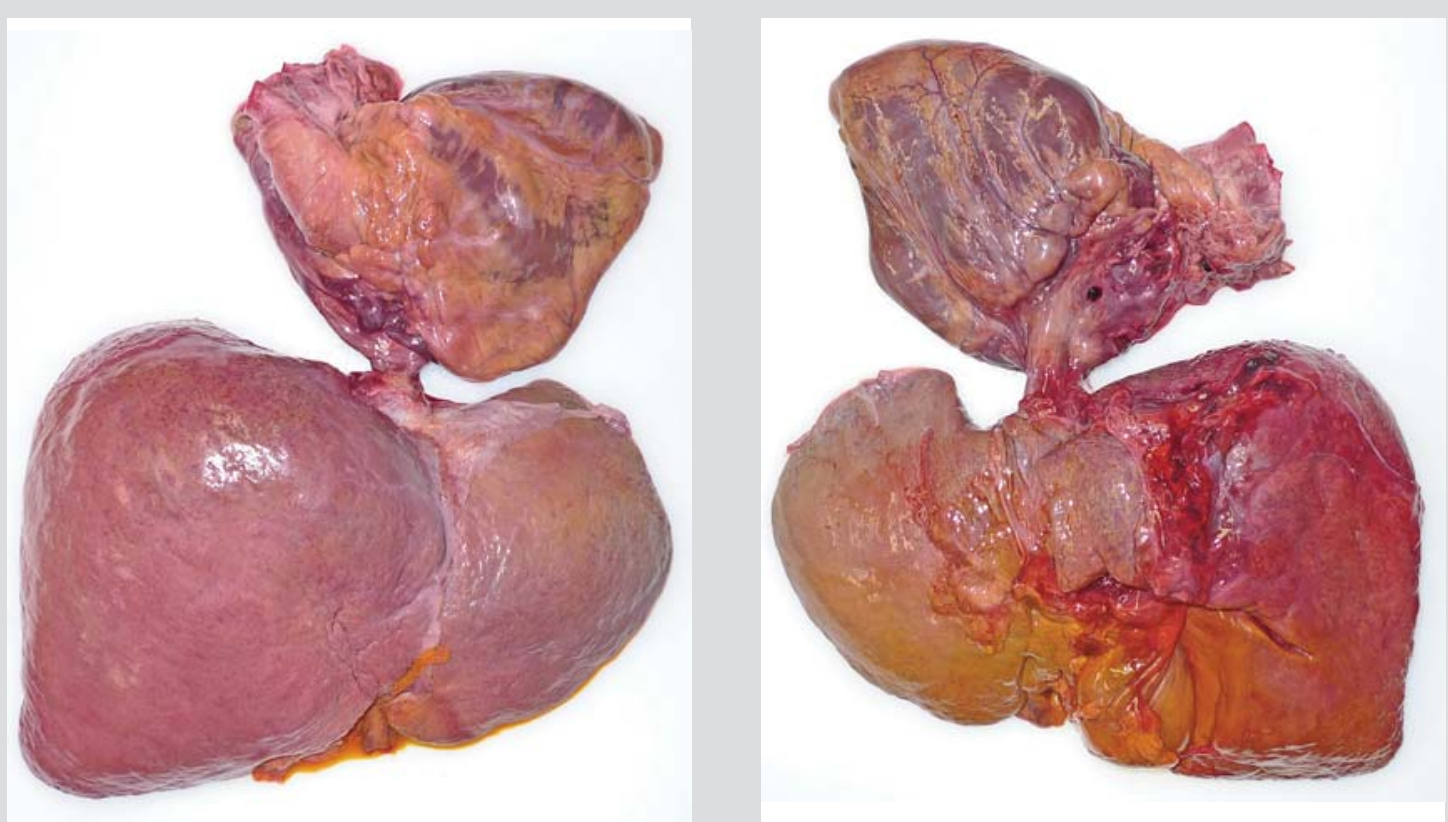

Figuras 1 y 2.

\section{Bibliografía}

1. Shah NL, Shanley CJ, Prince MR, Wakefield TW. Deep venous thrombosis complicating a congenital absence of the inferior vena cava. Surgery 1996;120:891-6.

2. Sakellaris G, Tilemis S, Papakonstantinou O, Bitsori $M$, Tsetis D, Charissis G. Deep venous thrombosis in a child associated with an abnormal inferior vena cava. Acta Paediatr. 2005;94:242-4.

3. Gayer G, Luboshitz J, Hertz M, Zissin R, Thaler M, Lubetsky A, et al. Congenital anomalies of the inferior vena cava revealed on CT in patients with deep vein thrombosis. AJR Am J Roentgenol. 2003; 180:729-32

4. Ruggeri M, Tosetto A, Castaman G, Rodeghiero F. Congenital absence of the inferior vena cava: a rare risk factor for idiopathic deep-vein thrombosis. Lancet 2010;357:441.

5. Awartani KA, McComb PF. Ovarian cyst formation and congenital absence of the inferior vena cava: case report. Clin Exp Obstet Gynecol. 1999;26:147-8.

6. Castro FJ, Pérez C, Narváez FJ, García A, Biosca M, Vilaseca J, et al. Agenesia de vena cava inferior como factor de riesgo de tromboembolismo pulmonar. An Med Interna (Madrid) [Internet] 2003;20:34-6.

7. Ramanathan T, Hughes TM, Richardson AJ. Perinatal inferior vena cava thrombosis and absence of the infrarenal inferior vena cava. J Vasc Surg. 2001;33:1097-9.

8. Siragusa S, Anastasio R, Falaschi F, Bonalumi G, Bressan MA. Congenital absence of inferior vena cava. Lancet 2001;26;357:1711.

9. Hoffman GH, Larson VO, Shipman GA, Sparger C. Venous anomaly of the hemiazygos system. Radiology 1961;77:626-8.

10. Ricci EP. Agenesia de la vena cava inferior. Rev Arg de Ultrasonido. 2009;8:132-3.

11. Mata Campos JE, Martínez Gámez FJ, Sánchez Mestre ML, Galán Zafra M, Maza Montero E. Abdomen agudo como forma atípica de una agenesia de la vena cava inferior: a propósito de un caso. Angiología 2008;60:61-5.

12. Over LM, van der Laan JG, Gökemeijer JD. Deep venous thrombosis as a complication of congenital absence of vena cava inferior. Ned Tijdschr Geneeskd. 2001;145:2280-3. 
13. Chuang VP, Mena CE, Hoskins PA. Congenital anomalies of the inferior vena cava. Review of the embriogenesis and presentation of a simplified classification. Br J Radiol. 1974;47:206-13.

14. Bass JE, Redwine MD, Kramer LA, Huynh PT, Harris JH. Spectrum of congenital anomalies of the inferior vena cava: sectional imaging findings. Radiographics 2000;20:639-52.

15. Edwards EA. Clinical anatomy of lesser variations of the inferior vena cava; and a proposal for classifying the anomalies of this vessel. Angiology 1951;2:85-99.

16. Minniti S, Visentini S, Procacci C. Congenital anomalies of the venae cavae: embryological origin, imaging features and report of three new variants. Eur Radiol. 2002;12:2040-55.

17. Aris A. Agenesia de la vena cava inferior. Angiología 1972;24:83-7.

18. Castro Iglesias AM, Diaz Bermudez J, Gago Ferreiro C, Noya Castro A. Vena cava inferior doble. Actas Urol Esp [online] 2010;34:823-4. ISSN 0210-4806.

19. Bussat L, Bopp P, Duchosal PW. Congenital heart disease with the Ivemark syndrome and absence of the inferior vena cava. Radiology 1965;84:657-9.

\section{Vi a jes $\mid$ Cajasol}

Contamos con un Departamento dedicado exclusivamente a la Organización Profesional de todo tipo de Eventos: Congresos, Simposios, Eventos Deportivos/Culturales, Reuniones/Encuentros, Cursos/Jornadas, etc.

Con los mejores y más modernos equipos de trabajo, personal altamente cualificado y experiencia demostrable. Avanzadas Tecnologías de Gestión de Congresos, con equipos informáticos especificos para la organización de eventos.

Reconocido prestigio empresarial, por planificación, viabilidad económica, gestión exclusiva, y disponibilidad territorial con logística para diseñar eventos en cualquier punto de España o Resto del Mundo.

Para nosotros, cada evento tiene que salir perfecto Contáctenos y compruébelo

www.viajescajasol.es

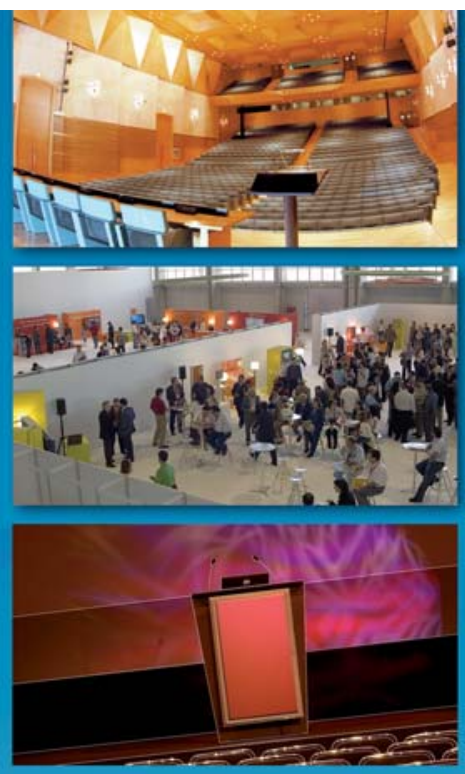

Oficina de Eventos: Santo Domingo de la Calzada, 5 41018 Sevilla 954981089 eventos@viajescajasol.es 